\title{
Crecimiento, producción y calidad en brócoli cultivado bajo diferentes dosis de abono nitrogenado
}

\author{
Growth, yield and quality in broccoli grown under different nitrogen fertilizer rates
}

\author{
David Risco Arias ${ }^{1 *}$, Alberto Cristóbal Gutiérrez Albán ${ }^{1}$ y Marcia Eduvijes Buenaño \\ Sánchez ${ }^{1}$ \\ ${ }^{1}$ Facultad de Ciencias Agropecuarias, Universidad Técnica de Ambato, Ecuador. \\ * Autor para correspondencia (da.risco@uta.edu.ec) \\ Recibido: 17/03/2016; Aceptado: 12/05/2016. \\ Doi:10.18004/investig.agrar.2016.junio.44-48
}

\begin{abstract}
RESUMEN
El brócoli es uno de los cultivos de mayor importancia económica en los Andes ecuatorianos. La fertilización nitrogenada afecta de manera directa a su producción, siendo uno de los nutrientes más importantes para las plantas. Con el objetivo de evaluar la respuesta del cultivo de brócoli al abono nitrogenado, se llevó a cabo el experimento durante el año 2014 con un diseño en bloques completos al azar, con cinco tratamientos y tres repeticiones. Los tratamientos fueron $200 \mathrm{~kg} \mathrm{ha}^{-1}\left(\mathrm{~N}_{200}\right)$, $250 \mathrm{~kg} \mathrm{ha}^{-1}\left(\mathrm{~N}_{250}\right), 300 \mathrm{~kg} \mathrm{ha}^{-1}\left(\mathrm{~N}_{350}\right), 350 \mathrm{~kg} \mathrm{ha}^{-1}\left(\mathrm{~N}_{350}\right)$ y $400 \mathrm{~kg} \mathrm{ha}^{-1}\left(\mathrm{~N}_{400}\right)$ de abono nitrogenado en forma de nitrato amónico (33\%). En las plantas se evaluó longitud y calibre del tallo, número de hojas, peso y diámetro de la pella en cosecha, producción por hectárea, eficiencia en el uso de nitrógeno y concentración de nutrientes. La longitud del tallo fue mayor para el tratamiento $\mathrm{N}_{400}$ a los 50 días después de la siembra, pero el crecimiento vegetativo no fue afectado por los tratamientos en la evaluación realizada a los 70 días después de la siembra. Por el contrario, en los parámetros de cosecha, el tratamiento $\mathrm{N}_{350}$ obtuvo la mayor producción en cosecha $\left(17,81 \mathrm{t} \mathrm{ha}^{-1}\right)$, superior a la del tratamiento $\mathrm{N}_{400}(14,02 \mathrm{t}$ $\mathrm{ha}^{-1}$ ). No obstante, el tratamiento $\mathrm{N}_{350}$ obtuvo las menores concentraciones de nutrientes como el potasio. El tratamiento $\mathrm{N}_{200}$ obtuvo la menor producción, pero realizó un uso más eficiente del nitrógeno. Este experimento pone de manifiesto los efectos adversos de un exceso de fertilización nitrogenada en la producción de brócoli y la importancia de hacer ensayos para diferentes condiciones climáticas y edafológicas.
\end{abstract}

Palabras clave: Brassica oleracea var. italica (L.), calidad, nitrógeno, potasio, producción.

\begin{abstract}
Broccoli is one of the most economically important crops in the Ecuadorian Andes. Nitrogen fertilization directly affects its production because it is one of the most important nutrient for plants. In order to evaluate broccoli crop response to nitrogen fertilizer, a study was carried out during 2014 in a randomized block design with five treatments and three replicates. The treatments were 200 $\mathrm{kg} \mathrm{ha}^{-1}\left(\mathrm{~N}_{200}\right), 250 \mathrm{~kg} \mathrm{ha}^{-1}\left(\mathrm{~N}_{250}\right), 300 \mathrm{~kg} \mathrm{ha}^{-1}\left(\mathrm{~N}_{350}\right), 350$ $\mathrm{kg} \mathrm{ha}^{-1}\left(\mathrm{~N}_{350}\right)$ and $400 \mathrm{~kg} \mathrm{ha}^{-1}\left(\mathrm{~N}_{400}\right)$ of nitrogen fertilizer in the form of ammonium nitrate $(33 \%)$. The following variables were evaluated: stem length and diameter, number of leaves, head weight and diameter at harvesting, total hectare yield, nitrogen use efficiency and nutrients concentration. Stem length was the highest in N400 treatment at 50 days after sowing, but vegetative growth was not affected by treatments at 70 days after sowing. Conversely, in the harvesting parameters, $\mathrm{N}_{350}$ treatment yielded the highest crop production $\left(17,81 \mathrm{t} \mathrm{ha}^{-1}\right)$, higher than that of the $\mathrm{N}_{400}$ treatment $\left(14,02 \mathrm{t} \mathrm{ha}^{-1}\right)$. However, treatment $\mathrm{N}_{350}$ scored lower concentrations of nutrients such as potassium. The $\mathrm{N}_{200}$ treatment yielded the lowest production but made a more efficient use of nitrogen. This experiment showed the adverse effects of an excess of nitrogen fertilization on broccoli production and the importance of carrying out assays for different climatic and soil conditions.
\end{abstract}

Keywords: Brassica oleracea var. italica (L.), quality, nitrogen, potassium, production. 


\section{INTRODUCCIÓN}

El brócoli (Brassica oleracea var. italica) es uno de los cultivos no tradicionales más importantes de los Andes ecuatorianos, debido principalmente a la demanda del producto en varios países a nivel mundial como Estados Unidos, Japón y Alemania. Pertenece a los cultivos de col (Familia Brassicaceae), y es un vegetal muy nutritivo, ya que su inflorescencia, que es el producto comercial, contiene entre otros nutrientes Ácido Ascórbico, Vitamina A, Calcio y otros componentes como flavonoides y glucosinolatos con efectos antioxidantes y anticancerígenos (Yoldas et al. 2008).

El nitrógeno es un macronutriente esencial para las plantas y juega un papel principal en el crecimiento y desarrollo de los cultivos. Es componente de muchos compuestos estructurales, genéticos y metabólicos como aminoácidos, ácidos nucleicos y clorofila. La nutrición nitrogenada afecta de manera significativa a la producción y la calidad de los cultivos y es reconocido como el nutriente más limitante para el desarrollo vegetal (López Berenguer et al. 2007).

Como muchos otros cultivos, el brócoli necesita grandes cantidades de nutrientes para conseguir una óptima producción (Gutezeit 2004). Los agricultores normalmente tienden a aplicar dosis elevadas de fertilizante nitrogenado con el objetivo de obtener una mayor producción, pero un exceso puede dar lugar a la contaminación por lixiviación de nitratos y a una reducción en la producción y en la calidad del brócoli (Moniruzzaman et al. 2007). Estas son razones importantes por las que es necesario mejorar la eficiencia en el uso del abono nitrogenado en cultivos vegetales.

Los efectos de la fertilización nitrogenada en la producción y la calidad del brócoli necesitan ser mejor estudiados debido a que existen diferencias muy amplias entre las recomendaciones actuales. Bakker et al. (2009) recomendaron una dosis de aproximadamente $180 \mathrm{~kg} \mathrm{ha}^{-1}$ de abono nitrogenado para obtener una óptima producción. Por otro lado, Erdem et al. (2010) informaron que la dosis más efectiva de abono nitrogenado en brócoli es de $250 \mathrm{~kg} \mathrm{ha}^{-1}$, mientras que Castellanos et al. (2001) obtuvieron la mayor producción con $400 \mathrm{~kg} \mathrm{ha}^{-1}$ de abono nitrogenado.

El objetivo del presente estudio fue optimizar la nutrición nitrogenada en brócoli para las condiciones climáticas y de suelo de la provincia de Tungurahua, Ecuador.

\section{MATERIALES Y MÉTODOS}

El ensayo se realizó durante los meses de abril a junio de 2014 en el Campus Querochaca de la Universidad Técnica de Ambato, Ecuador ( $01^{\circ} 22^{\prime}$ S, 78 $38^{\circ}$ ' O, 2865 msnm).

El suelo de la zona de estudio se clasifica como Andisol (SSS 2006), son derivados de cenizas volcánicas, de textura franco arenosa y caracterizados por una baja densidad aparente, una alta porosidad, una alta concentración de materia orgánica y baja de nitrógeno. Las propiedades químicas del suelo antes del ensayo fueron: pH 6,65, concentración de materia orgánica 2,8\%, Nitrógeno disponible 26,3 ppm, Fósforo disponible 13,9 ppm y Potasio disponible $1,1 \mathrm{cmol} \mathrm{kg}^{-1}$.

El clima de la zona de estudio es ecuatorial de alta montaña, caracterizado por una alta variación diaria de la temperatura y una baja variación estacional (Pourrut et al. 1995). Las condiciones climáticas durante el experimento fueron medidas en una estación climática situada en el Campus Querochaca (Figura 1). Durante el desarrollo del cultivo la temperatura máxima media fue de $17,9^{\circ} \mathrm{C}$, la temperatura mínima media de $8,7^{\circ} \mathrm{C}$ y la precipitación total de $213,3 \mathrm{~mm}$.

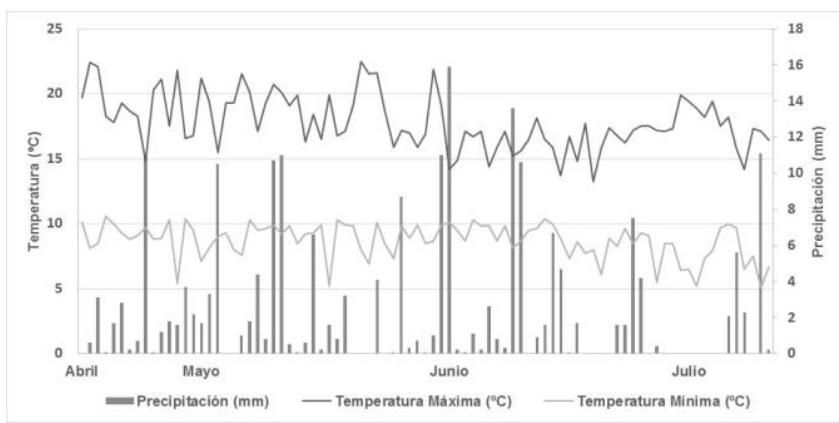

Figura 1. Condiciones climáticas durante el ciclo de cultivo.

El ensayo se realizó mediante un diseño experimental en bloques completos al azar que comprendió cinco tratamientos $\mathrm{y}$ tres repeticiones. Los tratamientos consistieron en cinco niveles de fertilizante nitrogenado: $\mathrm{N}_{200}\left(200 \mathrm{~kg} \mathrm{ha}^{-1}\right), \mathrm{N}_{250}\left(250 \mathrm{~kg} \mathrm{ha}^{-1}\right), \mathrm{N}_{300}\left(300 \mathrm{~kg} \mathrm{ha}^{-1}\right)$, $\mathrm{N}_{350}\left(350 \mathrm{~kg} \mathrm{ha}^{-1}\right), \mathrm{N}_{400}\left(400 \mathrm{~kg} \mathrm{ha}^{-1}\right)$, añadido en forma de nitrato amónico (33\%). El fertilizante fue aplicado en dos fases, la primera el día de siembra y la segunda 30 días después de la siembra (DDS).

Cada unidad experimental constaba de 28 plantas de brócoli cv. Mónaco a una distancia de plantación de 40 x 
$40 \mathrm{~cm}$. Todas las medidas de crecimiento vegetativo y parámetros de cosecha fueron tomadas en las 10 plantas centrales mientras que las 18 plantas restantes fueron consideradas como plantas guarda. Las plantas de brócoli fueron sembradas en campo tras 30 días en semillero, cuando habían desarrollado 3 o 4 hojas, el 16 de abril y la cosecha tuvo lugar el 11 de junio. Las operaciones de cultivo generales así como la protección de las plantas se realizaron conforme a las necesidades del cultivo.

En las plantas seleccionadas se tomaron las siguientes medidas: longitud y calibre del tallo (50, 70 DDS), número de hojas $(50,70 \mathrm{DDS})$ y peso y diámetro de la pella en cosecha (87 DDS). La eficiencia en el uso del nitrógeno (EUN) fue calculada como los kilogramos de producción comercial (pella) divididos entre los kilogramos de fertilizante nitrogenado aplicados (Castellanos et al. 2001). En cosecha, se recolectaron cuatro pellas por parcela experimental, se pesaron, se secaron en estufa a $105^{\circ} \mathrm{C}$ durante $24 \mathrm{~h}$ y se volvieron a pesar para calcular el contenido de humedad. En estas mismas plantas se calculó la concentración de nitrógeno total mediante el método Kjeldahl (AOAC 1990), y la concentración de calcio, magnesio y potasio, mediante digestión vía húmeda y lectura en espectrómetro de absorción atómica (AOAC 1990).

El análisis estadístico de los datos se realizó con el programa Infostat (Di Rienzo et al. 2014). En primer lugar se realizó un análisis de varianza y posteriormente se utilizó el test de Tukey para la comparación de medias entre los diferentes tratamientos.

\section{RESULTADOS Y DISCUSIÓN}

En cuanto al crecimiento vegetativo, la longitud del tallo fue superior para el tratamiento $\mathrm{N}_{400}$ con respecto a $\mathrm{N}_{300}$ a los 50 DDS (Tabla 1). A los 70 DDS, el valor máximo de los parámetros vegetativos (longitud y diámetro del tallo y número de hojas) se obtuvo en el tratamiento $\mathrm{N}_{400}$ a 70 DDS (Tabla 1), aunque las diferencias no fueron estadísticamente significativas. De acuerdo con Hussain et al. (2012) una mayor cantidad de aplicación de nitrógeno en brócoli dio lugar a un mejor crecimiento vegetativo como se observó en la longitud y calibre del tallo. El nitrógeno es uno de los nutrientes más importantes para el crecimiento de las plantas al ser componente estructural de las principales biomoléculas de la materia vegetal, como proteínas y ácidos nucleicos.

Tabla 1. Desarrollo vegetativo de los diferentes niveles de abono nitrogenado sobre brócoli. Tungurahua, Ecuador,

\begin{tabular}{|c|c|c|c|c|c|c|}
\hline \multirow[t]{2}{*}{ Tratamiento } & \multicolumn{2}{|c|}{ Longitud tallo $(\mathrm{cm})$} & \multicolumn{2}{|c|}{ Calibre tallo $(\mathrm{cm})$} & \multicolumn{2}{|c|}{ Número de hojas } \\
\hline & $50 * \mathrm{DDS}$ & 70 DDS & $50 \mathrm{DDS}$ & $70 \mathrm{DDS}$ & $50 \mathrm{DDS}$ & $70 \mathrm{DDS}$ \\
\hline $\mathrm{N}_{200}$ & $10,4 \mathrm{ab}$ & $15,6 \mathrm{a}$ & $1,6 \mathrm{a}$ & $3,0 \mathrm{a}$ & $9,0 \mathrm{a}$ & $14,0 \mathrm{a}$ \\
\hline $\mathrm{N}_{250}$ & $10,0 \mathrm{ab}$ & $15,8 \mathrm{a}$ & $1,6 \mathrm{a}$ & $3,0 \mathrm{a}$ & $9,0 \mathrm{a}$ & $14,0 \mathrm{a}$ \\
\hline $\mathrm{N}_{300}$ & $9,9 \quad b$ & $15,9 \mathrm{a}$ & $1,5 \mathrm{a}$ & $2,9 \mathrm{a}$ & $9,0 \mathrm{a}$ & $14,0 \mathrm{a}$ \\
\hline $\mathrm{N}_{350}$ & $10,5 \mathrm{ab}$ & $15,9 \mathrm{a}$ & $1,7 \mathrm{a}$ & $3,1 \mathrm{a}$ & $9,0 \mathrm{a}$ & $14,0 \mathrm{a}$ \\
\hline $\mathrm{N}_{400}$ & $10,8 \mathrm{a}$ & $16,5 \mathrm{a}$ & $1,6 \mathrm{a}$ & $3.2 \mathrm{a}$ & $9,0 \mathrm{a}$ & $15,0 \mathrm{a}$ \\
\hline E. $E^{1}$. & 0,1 & 0,15 & 0,03 & 0,04 & 0,08 & 0,09 \\
\hline C. $V^{2}$. & 0,04 & 0,02 & 0,04 & 0,04 & 0,00 & 0,03 \\
\hline Probabilidad & 0,02 & 0,42 & 0,33 & 0,38 & 0,22 & 0,21 \\
\hline
\end{tabular}

*DDS: Días después de la siembra. Misma letra en columnas indica que no existen diferencias significativas para Análisis de Varianza y Test de Tukey al 5\%. ${ }^{1}$ E. E.: Error estándar. ${ }^{2}$ C.V.: Coeficiente de variación (\%).

Por el contrario, los parámetros de cosecha no siguieron el mismo comportamiento que los parámetros vegetativos. El peso y diámetro de la pella en cosecha y la producción final fueron significativamente mayores en el tratamiento $\mathrm{N}_{350}$ que en el tratamiento $\mathrm{N}_{400}$ (Tabla 2). La producción en cosecha fue un $27 \%$ superior en $\mathrm{N}_{350}$ que en $\mathrm{N}_{400}$. Este resultado contradice a diversas investigaciones en las que la mayor dosis de fertilizante nitrogenado dio lugar a unos parámetros de producción superiores (Erdem et al. 2010,
Kumar et al. 2013, Vagen et al. 2007). En estos estudios, los valores de abono nitrogenado variaron entre 0 y 250 $\mathrm{kg} \mathrm{ha}^{-1} \mathrm{y}$ el nivel óptimo de nitrógeno pudo ser mayor al evaluado, como ocurrió en el presente estudio, donde la mayor producción se obtuvo con una dosis de abono de $350 \mathrm{~kg} \mathrm{ha}^{-1}$. Los resultados de este trabajo fueron superiores en $40 \mathrm{~kg} \mathrm{ha}^{-1}$ a los $310 \mathrm{~kg} \mathrm{ha}^{-1}$ recomendados para el cultivo de brócoli en Alemania (Feller y Fink 2000) y confirman el hecho de que una mayor cantidad de 
fertilizante nitrogenado puede ser perjudicial no solo para cultivo. el medio ambiente sino también para la producción del

Tabla 2. Parámetros de cosecha de las distintas dosis de fertilizante nitrogenado sobre brócoli. Tungurahua, Ecuador, 2014.

\begin{tabular}{cllll}
\hline Tratamiento & Peso pella $(\mathrm{kg})$ & Diámetro pella $(\mathrm{cm})$ & Producción $\left(\mathrm{t} \mathrm{ha}^{-1}\right)$ & EUN $^{1}(\mathrm{~kg} \mathrm{pella} / \mathrm{kg} \mathrm{N})$ \\
\hline $\mathrm{N}_{200}$ & $0,23 \mathrm{~b}$ & $11,49 \mathrm{~b}$ & $14,45 \mathrm{~b}$ & $72,27 \mathrm{a}$ \\
$\mathrm{N}_{250}$ & $0,21 \mathrm{~b}$ & $11,60 \mathrm{ab}$ & $13,20 \mathrm{~b}$ & $52,79 \mathrm{~b}$ \\
$\mathrm{~N}_{300}$ & $0,20 \mathrm{~b}$ & $12,00 \mathrm{ab}$ & $12,66 \mathrm{~b}$ & $42,19 \mathrm{bc}$ \\
$\mathrm{N}_{350}$ & $0,29 \mathrm{a}$ & $12,77 \mathrm{a}$ & $17,81 \mathrm{a}$ & $50,89 \mathrm{~b}$ \\
$\mathrm{~N}_{400}$ & $0,22 \mathrm{~b}$ & $11,56 \mathrm{~b}$ & $14,02 \mathrm{~b}$ & $35,17 \mathrm{c}$ \\
E.E & 0,01 & 0,14 & 0,37 & 1,49 \\
C. $\mathrm{V}^{3}$. & 0,15 & 0,05 & 0,14 & 0,28 \\
Probabilidad & $<0,01$ & 0,02 & $<0,01$ & $<0,01$ \\
\hline
\end{tabular}

Misma letra en columnas indica que no existen diferencias significativas para Análisis de Varianza y Test de Tukey al 5\%. ${ }^{1}$ EUN: Eficiencia en el uso del nitrógeno, E. E.: ${ }^{2}$ Error estándar. ${ }^{3}$ C.V.: Coeficiente de variación (\%).

La eficiencia en el uso del nitrógeno (Tabla 2) fue mayor para el tratamiento con la menor dosis $\left(\mathrm{N}_{200}\right)$ y fue menor en el tratamiento con mayor dosis $\left(\mathrm{N}_{400}\right)$. Los agricultores tienden a aplicar cantidades excesivas de fertilizante nitrogenado a los cultivos, pero una reducción en la dosis de abono de $400 \mathrm{~kg} \mathrm{ha}^{-1}$ a $350 \mathrm{~kg} \mathrm{ha}^{-1}$ permitió un aumento en la producción además de una mejor eficiencia en el uso de nitrógeno para las condiciones de clima y suelo de los Andes ecuatorianos.

La calidad nutricional no se vio afectada de forma significativa por los diferentes niveles de abono nitrogenado excepto en el caso de la concentración de potasio (Tabla 3) donde el tratamiento $\mathrm{N}_{350}$ obtuvo menor concentración que el tratamiento $\mathrm{N}_{400}$ pero sin diferir de las demás dosis. El aumento en la dosis de fertilizante nitrogenado no provocó diferencias en el contenido de humedad de la pella como observó El-Helaly (2012). En cuanto a la concentración de nitrógeno, a diferencia de otros estudios donde la absorción de nitrógeno aumentaba (Fabek et al. 2012) o disminuía (El-Helaly 2012) con aumentos en la dosis de abono nitrogenado, en esta investigación los resultados no mostraron una relación entre los niveles de fertilizante nitrogenado y la concentración en plantas de brócoli. De forma general, y sin obtener diferencias significativas, el tratamiento con mayor producción $\left(\mathrm{N}_{350}\right)$ tuvo una menor concentración de nutrientes, al igual que observaron Magd et al. (2010).

Tabla 3. Composición nutritiva de la pella de brócoli afectada por diferentes niveles de abono nitrogenado. Tungurahua, Ecuador, 2014.

\begin{tabular}{clllll}
\hline Tratamiento & Humedad $(\%)$ & Nitrógeno $(\%)$ & Potasio $(\%)$ & Calcio $(\%)$ & Magnesio $(\%)$ \\
\hline N200 & $87,54 \mathrm{a}$ & $4,43 \mathrm{a}$ & $2,50 \mathrm{~b}$ & $0,37 \mathrm{a}$ & $0,25 \mathrm{a}$ \\
N250 & $86,79 \mathrm{a}$ & $3,90 \mathrm{a}$ & $2,49 \mathrm{~b}$ & $0,27 \mathrm{a}$ & $0,17 \mathrm{a}$ \\
N300 & $86,88 \mathrm{a}$ & $3,62 \mathrm{a}$ & $3,01 \mathrm{ab}$ & $0,27 \mathrm{a}$ & $0,20 \mathrm{a}$ \\
N350 & $86,70 \mathrm{a}$ & $3,21 \mathrm{a}$ & $2,68 \mathrm{~b}$ & $0,32 \mathrm{a}$ & $0,21 \mathrm{a}$ \\
N400 & $87,49 \mathrm{a}$ & $4,57 \mathrm{a}$ & $3,50 \mathrm{a}$ & $0,33 \mathrm{a}$ & $0,23 \mathrm{a}$ \\
E.E.1 & 0,17 & 0,19 & 0,11 & 0,02 & 0,01 \\
C.V.2 & 0,01 & 0,14 & 0,15 & 0,14 & 0,14 \\
Probabilidad & 0,45 & 0,09 & $<0,01$ & 0,21 & 0,34 \\
\hline
\end{tabular}

Misma letra en columnas indica que no existen diferencias significativas para Análisis de Varianza y Test de Tukey al $5 \%$. ${ }^{1}$ E. E.: Error estándar. C.V.: ${ }^{2}$ Coeficiente de variación (\%).

\section{CONCLUSIONES}

Las recomendaciones de fertilización nitrogenada en brócoli para obtener una mayor producción, según este estudio, son de $350 \mathrm{~kg} \mathrm{ha}^{-1}$ en las condiciones de clima y suelo de los Andes ecuatorianos. Los resultados de este trabajo ponen de manifiesto la importancia de realizar investigaciones de campo para diferentes condiciones climáticas y edafológicas, y la necesidad de programas eficientes de fertilización. 


\section{AGRADECIMIENTOS}

Esta investigación fue realizada bajo el proyecto 1246 CU - P - 2014 de la Universidad Técnica de Ambato (Ecuador). Los autores quieren agradecer a los operarios agrícolas del Campus Querochaca por su ayuda en los trabajos de campo.

\section{REFERENCIAS BIBLIOGRAFICAS}

AOAC (Association of Official Analytical Chemists, US). 1990. Official Methods of Analysis. 16 ed. Virginia, US, AOAC. 684 p.

Bakker, CJ; Swanton CJ; McKeown, AW. 2009. Broccoli growth in response to increasing rates of pre-plant nitrogen: II Dry matter and nitrogen accumulation. Canadian Journal of Plant Science 89(3):539-548.

Castellanos, JZ; Villalobos, S; Delgado, JA; MuñozRamos, J; Sosa, A; Vargas, P; Lazcano, I; ÁlvarezSánchez, E; Enríquez, SA. 2001. Use of best management practices to increase nitrogen use efficiency and protect environmental quality in a broccoli-corn rotation of Central Mexico. Communications in Soil Science and Plant Analysis 32(7\&8):1265-1292.

Di Rienzo, J; Casanoves, F; Balzarini, MG; González, L; Tablada, M; Robledo, CW. 2014. Infostat versión 2014 (en línea). AR. Consultado 27 may. 2015. Disponible en http://www.Infostat.com.ar.

El-Helaly, MA. 2012. Effect of nitrogen fertilization rates and potassium sources on broccoli yield, quality and storability. Research Journal of Agriculture and Biological Sciences 8(4):385-394.

Erdem, T; Arin, L; Erdem, Y; Polat, S; Deveci, M; Okursoy, H; Gültas, HT. 2010. Crop water stress index for assessing irrigation scheduling of dripirrigated broccoli (Brassica oleracea L. var. italica). Agricultural Water Management 97(5):681-688.

Fabek, S; Toth, N; Redovnikovic, IR; Custic, MH; Benko, B; Zutic, I. 2012. The effect of nitrogen fertilization on nitrate accumulation, and the content of minerals and glucosinolates in broccoli cultivars Food Technology and Biotechnology 50(2):183-191.

Feller, C; Fink, M. 2000. Nmin target values for field vegetables. Acta Horticulturae 571:195-201.
Gutezeit, B. 2004. Yield and nitrogen balance of broccoli at different soil moisture levels. Irrigation Science 23: 21-27.

Hussain, MJ; Sirajul Karim; AJ, M; Solaiman, ARM; Haque, MM. 2012. Effects of nitrogen and boron on the yield and hollow stem disorder of broccoli (Brassica oleracea var. italica). The Agriculturists 10(2):36-45

Kumar, M; Das, B; Prasad, KK; Kumar, P. 2013. Effect of integrated nutrient management on growth and yield of broccoli (Brassica oleracea var. italica) under Jharkhand conditions. Vegetable Science 40(1):117120.

López-Berenguer, C; Carvajal, M; Garcea-Viguera, C; Alcaraz, CF. 2007. Nitrogen, phosphorus, and sulfur nutrition in broccoli plants grown under salinity. Journal of Plant Nutrition 30:1855-1870.

Magd, E; Sawan, MMA; Zaki, OM. 2010. Productivity and quality of two broccoli cultivars as affected by different levels of nitrogen fertilizers. Australian Journal of Basic and Applied Sciences 4(12):61256133.

Moniruzzaman, M; Rahman, SML; Kibria, MG; Rahman, MA; Hossain, MM. 2007. Effect of boron and nitrogen on yield and hollowstem of broccoli. Journal of Plant Nutrition 1(3):24-29.

Pourrut, P; Gómez, G; Bermeo, A; Segovia, A. 1995. Factores condicionantes de los regímenes climáticos e hidrológicos. In Pourrut, P. Estudios de geografía: el agua en el Ecuador: clima, precipitaciones, escorrentía. Quito, EC, Corporación Editora Nacional. p. 7-12.

SSS (Soil Survey Staff, US). 2006. Keys to soil taxonomy. 10 ed. Washington D.C, US, USDA. 332 p.

Vagen, IM; Aamlik, TS; Skjelag, AO. 2007. Nitrogen fertilization to broccoli cultivars at different planting times: yield and nitrogen use. Acta Agriculturae Scandinavica 57:35-44.

Yoldas, F; Ceylan, S; Yagmur, B; Mordogan, N. 2008. Effects of nitrogen fertilizer on yield quality and nutrient content in broccoli. Journal of Plant Nutrition 31:1333-1343. 